\title{
Comparison of Crystal Violet Staining Assay and XTT Methods in the Evaluation of Biofilm Formation in Candida parapsilosis Candidemia Isolates
}

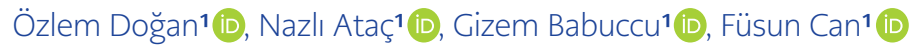 \\ 1 Department of Medical Microbiology, Koç University School of Medicine, İstanbul, Turkey
}

\begin{abstract}
Objective: Biofilm formation is one of the most important virulence factors of Candida species which leads to permanent infection foci by adhering to foreign materials and which are difficult to treat. Candida parapsilosis, which is one of the most common causes of candidemia in our country, is frequently isolated as a causative agent in catheterrelated infections. The most commonly used methods for evaluating the biofilm formation of Candida species are measuring cell viability with XTT (2,3-bis (2-methoxy-4-nitro-5sulfophenyl)-5-[(phenylamino) carbonyl]-2H-tetrazolium hydroxide) and evaluating the total biofilm mass with crystal violet (CV). The aim of this study is to evaluate the biofilm formation ability of C. parapsilosis candidemia isolates by XTT and (CV) methods and compare these methods with each other.

Materials and Methods: C. parapsilosis isolates sent from various hospitals between 2015 and 2019 were included in the study retrospectively, and the species-level identification was performed using the matrix- assisted laser desorption/ionization time-of-flight mass spectrometry (MALDI-TOF/MS) The biofilm formation of the isolates was compared based on the optical density (OD) values obtained by crystal violet and XTT methods. The biofilm formation of the isolates was evaluated by categorizing them into low, medium and high biofilm groups as $\pm 20 \%$ according to the median value of all strains.

Results: Totally, 79 C. parapsilosis candidemia isolates were included in this study and categorical compatibility between CV and XTT methods in low, medium and high biofilm groups was found as $69.6 \%, 60.6 \%$ and $73.9 \%$, respectively. The OD values of the XTT method in the high biofilm group were found statistically significantly higher when compared with the values from the CV method.

Conclusion: The compatibility of XTT and crystal violet methods in terms of biofilm measurement in C. parapsilosis isolates was considered acceptable, and no major variations were detected between the categories. According to these results, when evaluating the biofilm levels of C. parapsilosis isolates, high OD values obtained by the XTT method should be confirmed with the CV method.
\end{abstract}

Keywords: C. parapsilosis, candidemia, biofilm
Corresponding Author:

Özlem Doğan

E-mail:

ozldogan@ku.edu.tr

Received: November 26, 2021

Accepted: Arallk 24, 2021

Published: December 30, 2021

\section{Suggested citation:}

Doğan Ö, Ataç N, Babuccu G, Can F. Comparison of crystal violet

staining assay and XTT methods in the evaluation of biofilm formation in Candida parapsilosis candidemia isolates. Infect Dis Clin Microbiol 2021; 3: 158-163.

DOI: 10.36519/idcm.2021.104 


\section{INTRODUCTION}

$\mathrm{B}$ iofilm formation is one of the most important virulence factors for Candida species (1). Biofilm can be defined as a whole microbial community formed by microorganisms producing extracellular polymers and clustering in the matrix on various surfaces (2). Biofilm formation provides escape from host defence and also mediate the formation of resistance to antifungal drugs $(3,4)$. Candida species similar to other microorganisms also form biofilms on artificial surfaces (5). In Candida species, adhesion factors, biofilm formation and extracellular polymer synthesis are controlled by extremely complex cellular pathways. By transcriptomic studies revealing these pathways, transcription factors closely related to biofilm formation have been identified such as "biofilm and cell wall regulator 1 (Bcr1)", "enhanced filamentous growth protein 1(Efg-1)" and "transcription activator 1 (Тec-1)" (6).

Candidemia is the spread of Candida species from an endogenous or exogenous source, causing bloodstream infection. (7). Candida species cause recurrent candidemia with extremely high mortality by forming biofilms on surgical implants, central venous catheters or urinary catheters. (8). Catheter-related Candida infections cause important treatment difficulties in patients with immunosuppression, bone marrow or solid organ transplantation in clinics (9).

C. parapsilosis is the second most common cause of candidemia in Turkey after Candida albicans (10). C. parapsilosis which mostly causes infections in newborns and healthcare-associated outbreaks in hospitals is often isolated as a causative agent in catheter-related infections due to its ability to adhere to artificial surfaces and form "slime" (11). Therefore, evaluation of the biofilm-forming abilities of $C$. parapsilosis candidemia isolates has gained importance.

One of the most commonly used methods for the evaluation of biofilm formation in Candida species by phenotypic methods is the colorimetric method in which cell viability is measured using XTT (12). Another frequently used method in which biofilm formation is evaluated in Candida isolates is the total mass evaluation method with CV staining. Among these methods, living cells in the biofilm are detected with XTT, while the biofilm mass is evaluated as a whole in the CV staining method (13). The number of studies comparing these methods with each other is extremely limited, and generally, only one method is chosen in the studies. In addition, threshold values for these methods have not yet been determined and the methods have not been standardized yet (14). This study aims to evaluate the biofilm-forming abilities of C. parapsilosis candidemia isolates with XTT and CV methods to show the compatibility of these methods with each other.

\section{MATERIAL AND METHOD}

\section{Identification of Isolates and Biofilm Formation}

C. parapsilosis candidemia isolates that were sent to our laboratory from various hospitals between 2015 and 2019 were included in the study retrospectively. Species-level identification of the isolates was performed by VITEK ${ }^{\circledR}$ MS (bioMérieux, Marcy l'Etoile, France) matrix-assisted laser desorption ionization time-of-flight mass spectrometry

(MALDI-TOF/MS) microbial identification system at Koç University Hospital Microbiology Laboratory. The isolates were passaged from stocks kept at $-80^{\circ} \mathrm{C}$ to Sabouraud dextrose agar (SDA) (Becton, Dickinson and Company, USA) and were inoculated into RPMI medium (Sigma-Aldrich, USA, code: R6504) containing 2\% glucose and incubated for 24 hours at $200 \mathrm{rpm}$ and $37^{\circ} \mathrm{C}$. After incubation, $100 \mu \mathrm{l}$ of samples were adjusted to 0.5 McFarland density in RPMI medium with $2 \%$ glucose were transferred

\section{HIGHLIGHTS}

- In the evaluation of the biofilm formation in C. parapsilosis, the compatibility of $\mathrm{CV}$ and XTT methods is acceptable, and no major variations were detected.

- The OD values of the XTT method in the high biofilm group were found statistically significantly higher when compared with the values from the CV method.

- High optical density results obtained with the XTT method should be confirmed with CV assay. 
to sterile 96-well polystyrene plates. Plates were incubated for 24 hours at $75 \mathrm{rpm}$ and $37^{\circ} \mathrm{C}(13)$. Test medium without cells was added to the final well of each plate as the negative control.

\section{Biofilm Formation by Crystal Violet Method}

For the determination of the biofilm mass by the CV method, RPMI media with $2 \%$ glucose were removed from the wells after 24 hours of incubation, and after washing twice with phosphate-buffered saline (PBS), the plates were left to dry for 45 minutes. Then, $125 \mu \mathrm{l}$ of $1 \%$ crystal violet solution was added to the wells and incubated for 15 minutes at room temperature. Plates were washed twice with PBS and dried at room temperature for 30 minutes, then $200 \mu \mathrm{l}$ of $95 \%$ ethanol solution was added to the wells. Following the incubation of the plates for 15 minutes at room temperature, OD measurement was performed at $540 \mathrm{~nm}$ in the Multiskan GO (Thermo Fisher Scientific Inc., USA) spectrophotometer device. The absorbance values of the negative controls were subtracted from the values of the test wells to minimize background interference. Three different measurements were performed for each isolate, and standard deviation values were determined by taking the average of the results.

\section{Biofilm Formation by XTT Method}

After the biofilm formation, the wells were washed twice with PBS, and $100 \mu \mathrm{l}$ of XTT/menadione (Sigma-Aldrich, USA) (final concentration of $1 \mathrm{mM}$ ) was added to the samples and incubated at $37^{\circ} \mathrm{C}$ for two hours in the dark. After the incubation, the viability of the cells in the biofilm was determined by measuring the OD at $490 \mathrm{~nm}$ with the Multiskan GO spectrophotometer device. The absorbance values of the negative controls were subtracted from the values of the test wells to minimize background interference. Three different measurements were made for each isolate, and the standard deviation (SD) values were determined by taking the average of the results.

\section{Statistical Analysis}

The biofilm formation ability of the isolates was compared based on the OD values obtained by CV and XTT methods, and the biofilm formation level of the isolates was categorized as low, medium and high biofilm groups according to the median and $\pm 20 \%$ of all strains. The median OD value of all isolates was determined and \pm 20 of all isolates were accepted medium biofilm group (n:33) and the rest was calculated as low (n:23) and high (n:23) biofilm groups. When the methods were compared with each other, results in the same category (low, medium or high) with both methods were accepted as category agreement. For the statistical significance test, Tukey's multiple comparison test was applied following the one-way ANOVA test, and $p \leq 0.05$ was considered statistically significant.

\section{RESULTS}

In this study, 79 C. parapsilosis strains isolated from blood culture were included. All isolates were grouped with an average of $\pm 20 \%$ ( \pm 16 isolates) in order to evaluate biofilm formation according to the methods; 23 isolates were in the low, 33 isolates in the medium and 23 isolates in the high-level biofilm group. When the biofilm masses of all isolates were evaluated with CV staining method, the medi-

Table 1. Evaluation of biofilm level of C. parapsilosis isolates according to categories by crystal violet and XTT methods.

\begin{tabular}{|c|c|c|c|c|c|c|c|}
\hline \multicolumn{2}{|c|}{} & \multicolumn{2}{c|}{ CV } & \\
\hline Biofilm level & Number & Mean (OD nm) & Lowest (OD nm) & Highest (OD nm) & Mean (OD nm) & Lowest (OD nm) & Highest (OD nm) \\
\hline Low & 23 & 0.127 & 0.05 & 0.164 & 0.183 & 0.057 \\
\hline Medium & 33 & 0.348 & 0.177 & 0.546 & 0.291 & 0.299 \\
\hline High & 23 & 0.839 & 0.554 & 1.37 & 1.06 & 0.634 \\
\hline TOTAL & 79 & 0.427 & 0.05 & 1.37 & 0.561 & 0.399 \\
\hline
\end{tabular}


an biofilm value was $0.363 \mathrm{~nm}$ and the mean value was $0.427 \mathrm{~nm}(0.05-1.37 \mathrm{~nm}, \mathrm{SD}: 0.326)$. When the same isolates were evaluated with the XTT method in terms of biofilm density, the median biofilm value was $0.494 \mathrm{~nm}$, and the mean value was 0.561 $\mathrm{nm}$ (0.05-2.399 nm, SD: 0.426) (Table 1). The biofilm formation for each isolate was repeated three times and the mean standard deviation was determined as 0.08 with CV and 0.09 with XTT.

When the mean OD values of isolates were compared with each other by CV and XTT methods according to low (n:23), medium (n:33) and high (n:23) level biofilm formation groups; there was no statistically significant difference between the two methods in the low and medium groups ( $p>0.05$ ), but a statistically significant difference was found in the high biofilm group (p: 0.009) (Figure 1).

In the head-to-head comparison of the CV and XTT methods; 16 (69.6\%) of the 23 isolates measured in the low-level biofilm group by the CV method also formed low-level biofilms by the XTT, and the mean OD value of these isolates was determined as 0.170 and, the remaining seven isolates (30.4\%) formed a medium level of biofilm by XTT method and the mean OD value of these isolates was 0.402 . In the low-level biofilm group with the XTT method (mean OD value: 0.120$) 16$ of the 23 isolates were also formed low-level biofilms with the CV but seven of them (30.4\%) formed medium-level biofilm with the CV method. The category agreement was found to be $69.6 \%$ for isolates with low biofilm formation by both methods.

When medium-level biofilm groups were evaluated, 20 of the 33 isolates formed a medium-level of biofilm by both methods. Six isolates that were in the medium biofilm group by the CV method were included in the high-level biofilm group by the XTT method, and the mean OD value of these isolates measured by the XTT method was found to be 1.260. The six isolates that formed a medium level biofilm with the XTT method, formed a high-level biofilm with the CV method, and the mean OD value of these isolates is 0.648 . The category agreement of the two methods in terms of evaluating the medium level biofilm formation was determined as $60.6 \%$. While 17 of the 23 C. parapsilosis isolates that

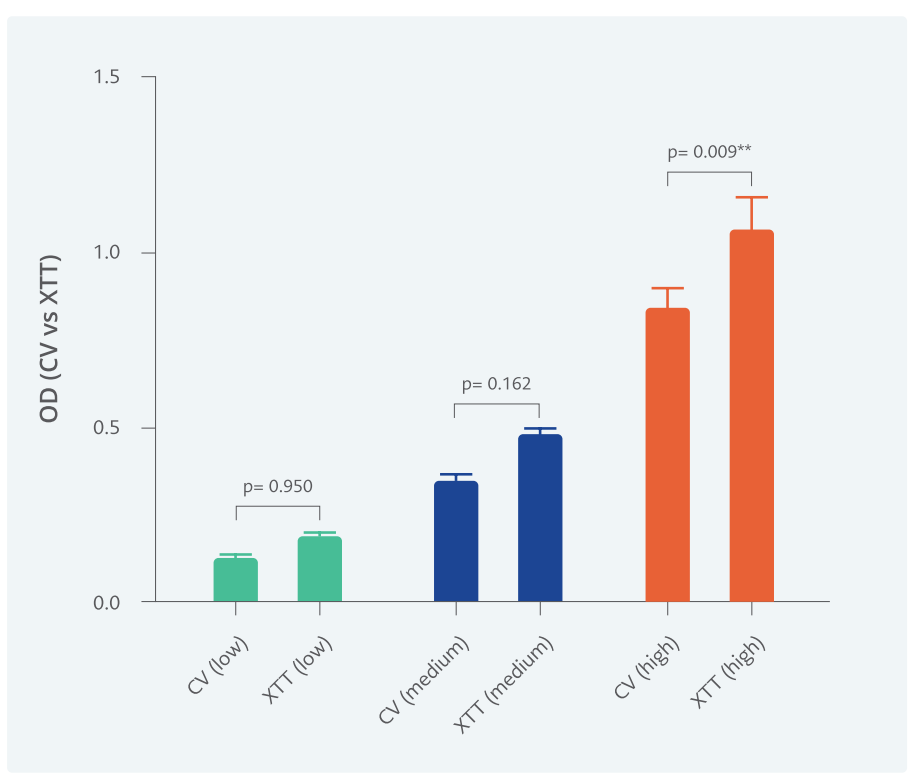

Figure 1. Comparison of the mean optical density values obtained by crystal violet (CV) and XTT methods according to the biofilm structure of all isolates (low, n=23; medium, n=33; high, $n=23$ ) **=statistically significant.

formed high biofilm were in the high-level biofilm group by both methods, but 6 isolates were detected as medium-level biofilms with each method. In the evaluation of biofilm formation of high-level biofilm-forming isolates, the category agreement of both methods was found to be $73.9 \%$.

\section{DISCUSSION}

Biofilm formation, which is one of the most important virulence factors of Candida species, leads to the formation of persistent foci and recurrent candidemia due to the adhesion to foreign surfaces, causing treatment failures with antifungal drugs (15). Biofilm formation has been associated with poor clinical outcomes, and mortality in Candida infections caused by high biofilm-forming isolates was found to be higher than low biofilm-forming isolates (16). In a study, infection with higher biofilm-forming Candida isolates, presence of central venous catheter and suboptimal dose of fluconazole were found to be independent risk factors for persistent candidemia (17). Considering the effect of biofilm formation on the follow-up and prognosis of patients in candidemia, there is an urgent need for an accurate, fast and standardized biofilm measurement method. 
There are many methods based on different techniques are used in the evaluation of biofilm formation of Candida species. One of the most commonly used methods for this purpose is the XTT method, in which cell viability is determined colorimetrically. This method is based on the conversion of XTT to formazan, an orange-colored compound, by mitochondrial succinoxidase, cytochrome P450 enzymes and flavoprotein oxidases in the presence of metabolic activity (12). Another frequently used method is the CV method and biomass measurement and in previous studies, these two methods have been used separately or together in the evaluation of biofilm formation in Candida species. In addition to these methods, the evaluation of the biofilm mass as a colony-forming unit (cfu) could be performed by separating the biofilm mass from the plastic surface by methods such as scraping, homogenization and sonication (18). Moreover, scanning electron microscopy or confocal microscopy are used in the evaluation of the density of the biofilm in centers where the infrastructure is sufficient. In the evaluation of biofilm formation in Candida species, the use of proteomic analyzes with the matrix-assisted laser desorption ionization time-of-flight mass spectrometry (MALDI-TOF MS) has come to the fore, and more successful results have been obtained in the demonstration of biofilm compared to standard methods (19).

The number of studies comparing the methods used in the evaluation of biofilm formation in Candida species is extremely limited. Marcos-Zambrano et al. compared the methods of biomass measurement with CV and metabolic activity measurement with the XTT method in the evaluation of biofilm formation in Candida fungemia isolates from blood cultures. They found that the agreement between the two methods was higher in C. albicans and C. parapsiosis isolates, but lower in C. tropicalis, C. krusei, C. glabrata species, and stated that both methods should be used together in determining the biofilm density (20). In our study, when we examined the agreement of both methods in C. parapsilosis candidemia isolates, the category agreement was found to be $73.9 \%, 60 \%$ and $69.6 \%$ in high, medium and low biofilm-forming isolates, respectively. When the categorical compatibility of C. parapsilosis isolates forming biofilms was evaluated in our study, the isolates detected in the high biofilm group with one of the methods were included in the high or medium biofilm group with the other method but were not included in the low biofilm group. Similarly, the isolates that were found to be in the low biofilm group with one method were also included in the low or medium group with the other method but were not in the high biofilm-forming group.

Although there was no major categorical change (transition from the low biofilm formation category to high group or from the high biofilm formation category to low group) between the two methods, higher mean $\mathrm{OD}$ values were detected in all three categories with the XTT method. In the high biofilm group, the OD values obtained by the XTT method were found to be statistically significantly higher when compared with CV. Zou et al. studied the biofilm-forming capacities of various Candida species with both XTT and CV methods, showing that C. parapsilosis was the highest biofilm-forming species and that the agreement of the two methods was acceptable in other Candida species except C. tropicalis and similar to the results of our study, higher $\mathrm{OD}$ values were determined by the XTT method in this study as well (21).

In our study, similar to previous studies, the compatibility of XTT and KV methods in terms of biofilm measurement was considered acceptable, and no major errors were detected between the categories. Higher OD values were determined by the XTT method in all isolates, and this difference was statistically significant in isolates with high biofilm. According to these results, when evaluating biofilm levels in C. parapsilosis isolates, the high OD values obtained by the XTT method should be confirmed by the CV method. The results of this study should be supported by larger studies involving different Candida species with a larger sample group, using different methods such as confocal microscopy and colony formation counting in addition to XTT and CV methods. 
Ethical Approval: The Biomedical Research Ethics Committee of Koç University approved the study on June 25, 2018, with the decision number of 165.IRB2.029-25-06-2018.

Informed Consent: N.A.

Peer-review: Externally peer-reviewed

Author Contributions: Concept - Ö.D., F.C.; Design - Ö.D., F.C.; Supervision - Ö.D., F.C., N.A.; Findings - Ö.D., F.C.; Materials - Ö.D.,
N.A., G.B., F.C.; Data Collection and/or Processing - Ö.D., N.A., G.B.; Analysis and/or Interpretation - Ö.D., G.B., F.C.; Literature Review Ö.D., G.B., F.C.; Writer - Ö.D., N.A., G.B., F.C.; Critical Reviews - Ö.D., F.C.

Conflict of Interest: The authors declare no conflict of interest.

Financial Disclosure: The authors declared that this study has received no financial support.

\section{REFERENCES}

1 Potera C. Forging a link between biofilms and disease. Science.1999;283(5409):1837-9. [CrossRef]

2 Donlan RM. Biofilm formation: a clinically relevant microbiological process. Clin Infect Dis. 2001;33(8):1387-92. [CrossRef]

3 Eix EF, Nett JE. How biofilm growth affects Candida-host interactions. Front Microbiol. 2020;11:1437. [CrossRef]

4 Mah TF, O'Toole GA. Mechanisms of biofilm resistance to an timicrobial agents. Trends Microbiol. 2001;9(1):34-9. [CrossRef]

5 Douglas LJ. Candida biofilms and their role in infection. Trends Microbiol. 2003;11(1):30-6. [CrossRef]

6 Cavalheiro M, Teixeira MC. Candida biofilms: Threats, challenges, and promising strategies. Front Med (Lausanne). 2018;5:28. [CrossRef

7 Antinori S, Milazzo L, Sollima S, Galli M, Corbellino M. Candidemia and invasive candidiasis in adults: A narrative review. Eur J Intern Med. 2016;34:21-8. [CrossRef]

8 Ramage G, Martínez JP, López-Ribot JL. Candida biofilms on implanted biomaterials: A clinically significant problem. FEMS Yeast Res. 2006;6(7):979-86. [CrossRef]

9 Hu B, Du Z, Kang Y, Zang B, Cui W, Qin B, et al. Catheter-related Candida bloodstream infection in intensive care unit patients: A subgroup analysis of the China-SCAN study. BMC Infect Dis. 2014;14:594. [CrossRef]

10 Arikan-Akdagli S, Gülmez D, Doğan Ö, Çerikçioğlu N, Doluca Dereli M, Birinci A, et al. First multicentre report of in vitro resistance rates in candidaemia isolates in Turkey. J Glob Antimicrob Resist. 2019;18:230-4. [CrossRef]

11 Tóth R, Nosek J, Mora-Montes HM, Gabaldon T, Bliss JM, Nosan chuk JD, et al. Candida parapsilosis: From genes to the bedside. Clin Microbiol Rev. 2019;32(2):e00111-18. [CrossRef]

12 Kuhn DM, Balkis M, Chandra J, Mukherjee PK, Ghannoum MA. Uses and limitations of the XTT assay in studies of Candida growth and metabolism. J Clin Microbiol. 2003;41(1):506-8. [CrossRef]

13 Melo AS, Bizerra FC, Freymüller E, Arthington-Skaggs BA, Colombo AL. Biofilm production and evaluation of antifungal susceptibility amongst clinical Candida spp. isolates, including strains of the Candida parapsilosis complex. Med Mycol. 2011;49(3):253-62. [CrossRef]

14 Bouza E, Guinea J, Guembe M. The Role of Antifungals against Candida Biofilm in Catheter-Related Candidemia. Antibiotics (Basel). 2014;4(1):1-17. [CrossRef]

15 Monfredini PM, Souza ACR, Cavalheiro RP, Siqueira RA, Colombo AL. Clinical impact of Candida spp. biofilm production in a cohort of patients with candidemia. Med Mycol. 2018;56(7):8038. [CrossRef]

16 Tumbarello M, Fiori B, Trecarichi EM, Posteraro P, Losito AR, De Luca A, et al. Risk factors and outcomes of candidemia caused by biofilm-forming isolates in a tertiary care hospital. PLoS One. 2012;7(3):e33705. [CrossRef]

17 Li WS, Chen YC, Kuo SF, Chen FJ, Lee CH. The impact of biofilm formation on the persistence of Candidemia. Front Microbiol. 2018;9:1196. [CrossRef]

18 Van Dijck P, Sjollema J, Cammue BP, Lagrou K, Berman J, d'Enfert $\mathrm{C}$, et al. Methodologies for in vitro and in vivo evaluation of efficacy of antifungal and antibiofilm agents and surface coatings against fungal biofilms. Microb Cell. 2018;5(7):300326. [CrossRef]

19 Aguiar PADF, Menezes RP, Penatti MPA, Moreira TA, Pimenta JP, Silva NBS, et al. Rapid detection of biofilm-producing Candida species via MALDI-TOF mass spectrometry. J Appl Microbiol. 2021. (Baskıda). [CrossRef]

20 Marcos-Zambrano LJ, Escribano P, Bouza E, Guinea J. Production of biofilm by Candida and non-Candida spp. isolates causing fungemia: Comparison of biomass production and metabolic activity and development of cut-off points. Int J Med Microbiol. 2014;304(8):1192-8. [CrossRef]

21 Zuo XS, Liu Y, Cai X, Zhan L, Hu K. Association of different Candida species with catheter-related candidemia, and the potential antifungal treatments against their adhesion properties and biofilm-forming capabilities. J Clin Lab Anal. 2021;35(4):e23738. [CrossRef] 